\title{
THE REVERSED HARDY-LITTLEWOOD-SOBOLEV TYPE INTEGRAL SYSTEMS WITH WEIGHTS
}

\author{
XIAOQIAN LIU
}

Abstract. This paper is concerned with the existence of positive entire solutions of a weighted integral system. Such a system comes from the conformal properties of the reversed HardyLittlewood-Sobolev inequality. Several sufficient conditions of the existence/nonexistence are presented.

Mathematics subject classification (2010): 26D15, 45E10, 45G05, 45M20.

Keywords and phrases: Reversed Hardy-Littlewood-Sobolev inequality, weighted Hardy-LittlewoodSobolev inequality, integral system, existence of positive solution.

\section{REFERENCES}

[1] J. Bebernes, Y. LEI, C. LI, A singularity analysis of positive solutions to an Euler-Lagrange integral system, Rocky Mountain J. Math., 41, (2011), 387-410.

[2] W. Chen, C. LI, The best constant in a weighted Hardy-Littlewood-Sobolev inequality, Proc. Amer. Math. Soc., 136, (2008), 955-962.

[3] L. Chen, Z. Liu, G. Lu, C. TAO, Reverse Stein-Weiss in-equalities and existence of their extremal functions, Trans. Amer.Math. Soc., 370, (2018), 8429-8450.

[4] W. Chen, C. LI, B. OU, Classification of solutions for an integral equation, Comm. Pure Appl. Math., 59, (2006), 330-343.

[5] J. Dou, M. Zhu, Reversed Hardy-Littlewood-Sobolev inequality, Internat. Math. Res. Notices, (2015), no. 19, 9696-9726.

[6] L. HUANG, On critical condition for a weighted integral system with negative exponents, Math. Inequal. Appl., 18, (2015), 1511-1517.

[7] C. Jin, C. LI, Symmetry of solutions to some systems of integral equations, Proc. Amer. Math. Soc., 134, (2006), 1661-1670.

[8] C. Jin, C. LI, Qualitative analysis of some systems of integral equations, Calc. Var. Partial Differential Equations, 26, (2006), 447-457.

[9] Y. LEI, On the integral systems with negative exponents, Discrete Contin. Dyn. Syst., 35, (2015), 1039-1057.

[10] Y. LEI, C. LI, Sharp criteria of Liouville type for some nonlinear systems, Discrete Contin. Dyn. Syst., 36, (2016), 3277-3315.

[11] Y. LEI, C. LI, C. MA, Asymptotic radial symmetry and growth estimates of positive solutions to weighted Hardy-Littlewood-Sobolev system, Calc. Var. Partial Differential Equations, 45, (2012), 4361.

[12] Y. LEI, Z. LÜ, Axisymmetry of locally bounded solutions to an Euler-Lagrange system of the weighted Hardy-Littlewood-Sobolev inequality, Discrete Contin. Dyn. Syst., 33, (2013), 1987-2005.

[13] Y. LEI, C. MA, Asymptotic behavior for solutions of some integral equations, Commun. Pure Appl. Anal., 10, (2011), 193-207.

[14] Y. LEI, C. MA, Optimal integrability of some system of integral equations, Front. Math. China, 9, (2014), 81-91.

[15] C. LI, J. LIM, The singularity analysis of solutions to some integral equations, Commun. Pure Appl. Anal., 6, (2007), 453-464. 
[16] Y. LI, Remark on some conformally invariant integral equations: the method of moving spheres, J. Eur. Math. Soc., 6, (2004), 153-180.

[17] E. LIEB, Sharp constants in the Hardy-Littlewood-Sobolev and related inequalities, Ann. of Math., 118, (1983), 349-374.

[18] Q. NGo, V. NGuYen, Sharp reversed Hardy-Littlewood-Sobolev inequality on $R^{n}$, Israel J. Math., 220, (2017), 1-35.

[19] M. ONODERA, On the shape of solutions to an integral system related to the weighted HardyLittlewood-Sobolev inequality, J. Math. Anal. Appl., 389, (2012), 498-510.

[20] E. Stein, G. Weiss, Fractional integrals in n-dimensional Euclidean space, J. Math. Mech., 7 , (1958), 503-514.

[21] X. XU, Uniqueness theorem for integral equations and its application, J. Funct. Anal., 247, (2007), 95-109.

[22] Y. ZHAO, Regularity and symmetry for solutions to a system of weighted integral equations, J. Math. Anal. Appl., 391, (2012), 209-222.

[23] W. ZIEMER, Weakly Differentiable Functions, Graduate Texts in Math. Vol. 120, Springer-Verlag, New York, 1989. 